\title{
THE EFFECTS OF VENOUS CONGESTION OF THE LIMBS UPON RENAL CLEARANCES AND THE EXCRETION OF WATER AND SALT. I. STUDIES IN NORMAL SUBJECTS AND IN HYPERTEN- SIVE PATIENTS BEFORE AND AFTER SPLANCHNICECTOMY ${ }^{1,2}$
}

\author{
BY ROBERT W. WILKINS, ${ }^{3}$ CLARENCE M. TINSLEY, ${ }^{4}$ JAMES W. CULBERTSON, ${ }^{5}$ \\ BELTON A. BURROWS, ${ }^{6}$ WALTER E. JUDSON, ${ }^{7}$ AND \\ CHARLES H. BURNETT 8

\begin{abstract}
(From the Robert Dawson Evans Memorial Department of Clinical Research and Preventive Medicine, Massachusetts Memorial Hospitals, and the Department of Medicine, Boston University School of Medicine, Boston, Mass.)
\end{abstract}

(Submitted for publication June 5, 1953; accepted July 8, 1953)

\section{INTRODUCTION}

The assumption of the upright position is known to cause decreases in urine volume, renal blood flow, and sodium excretion $(2,3)$. Since, by congesting the limbs of supine subjects, it is possible to simulate under better controlled conditions cer-

1 Presented in part at the Forty-First Annual Meeting of the American Society for Clinical Investigation, May 2, 1949 (1).

2 This investigation was supported in part by a grant from the National Heart Institute of the National Institutes of Health, U. S. P. H. S.

${ }^{3}$ Member, Evans Memorial, Massachusetts Memorial Hospitals; Visiting Physician, Massachusetts Memorial Hospitals ; Associate Professor of Medicine, Boston University School of Medicine.

4 Formerly Research Fellow in Medicine, Evans Memmorial, Massachusetts Memorial Hospitals; Assistant in Medicine, Boston University School of Medicine. Present Address: Stanford University School of Medicine, San Francisco, California.

5 Formerly Public Health Service Special Research Fellow of the National Heart Institute, Evans Memorial, Massachusetts Memorial Hospitals; Instructor in Medicine, Boston University School of Medicine. Present Address : University Hospitals, Iowa City, Iowa.

6 Associate Member, Evans Memorial, Massachusetts Memorial Hospitals; Associate Visiting Physician, Massachusetts Memorial Hospitals; Assistant Professor of Medicine, Boston University School of Medicine.

7 Assistant Member, Evans Memorial, Massachusetts Memorial Hospitals; Assistant Visiting Physician, Massachusetts Memorial Hospitals; Assistant Professor of Medicine, Boston University School of Medicine.

${ }^{8}$ Formerly Member, Evans Memorial, Massachusetts Memorial Hospitals; Visiting Physician, Massachusetts Memorial Hospitals; Associate Professor of Medicine, Boston University School of Medicine. Present Address: University of North Carolina School of Medicine, Chapel Hill, North Carolina. tain of the hemodynamic effects of the upright position while avoiding others, it seemed worthwhile to investigate the effects of this procedure upon renal clearances and the urinary excretion of salt and water. It seemed particularly pertinent to do so in hypertensive patients before and after lumbodorsal splanchnicectomy, an operation which conceivably might alter the renal responses to venous congestion. It also seemed relevant to inquire whether the renal disturbances caused by this procedure in any way resemble those found in association with other conditions characterized by venous congestion or edema in the limbs.

\section{SUBJECTS AND METHODS}

The subjects were patients admitted to the Massachusetts Memorial Hospitals, who at the time of study had either no cardiovascular-renal abnormalities (normotensive controls) or essential hypertension (hypertensives before and after splanchnicectomy). Throughout the test the subjects were given by mouth $200 \mathrm{cc}$. of water every half hour. Otherwise they were studied in the basal state, lying supine in bed. At the beginning of the test, blood pressure cuffs were applied smoothly to the upper thighs and bandaged (without compression) in place. A soft rubber catheter was placed in the bladder in a position that allowed complete drainage. A freshly prepared infusion containing 4 per cent glucose solution in water and appropriate amounts of inulin and sodium para-aminohippurate for renal clearance studies (4) was given at a rate of $4 \mathrm{cc}$. per minute in an antecubital vein. An indwelling needle, fitted with obturator for taking blood samples, was inserted through the skin anesthetized with metycaine into the brachial artery, or in a few experiments into a peripheral vein. Arterial pressure and pulse rate were measured by the usual clinical methods in the opposite arm. Accurately measured collections of urine were made and the bladder was washed with $20 \mathrm{cc}$. distilled water at (usually) 10-minute intervals and when desired, samples 


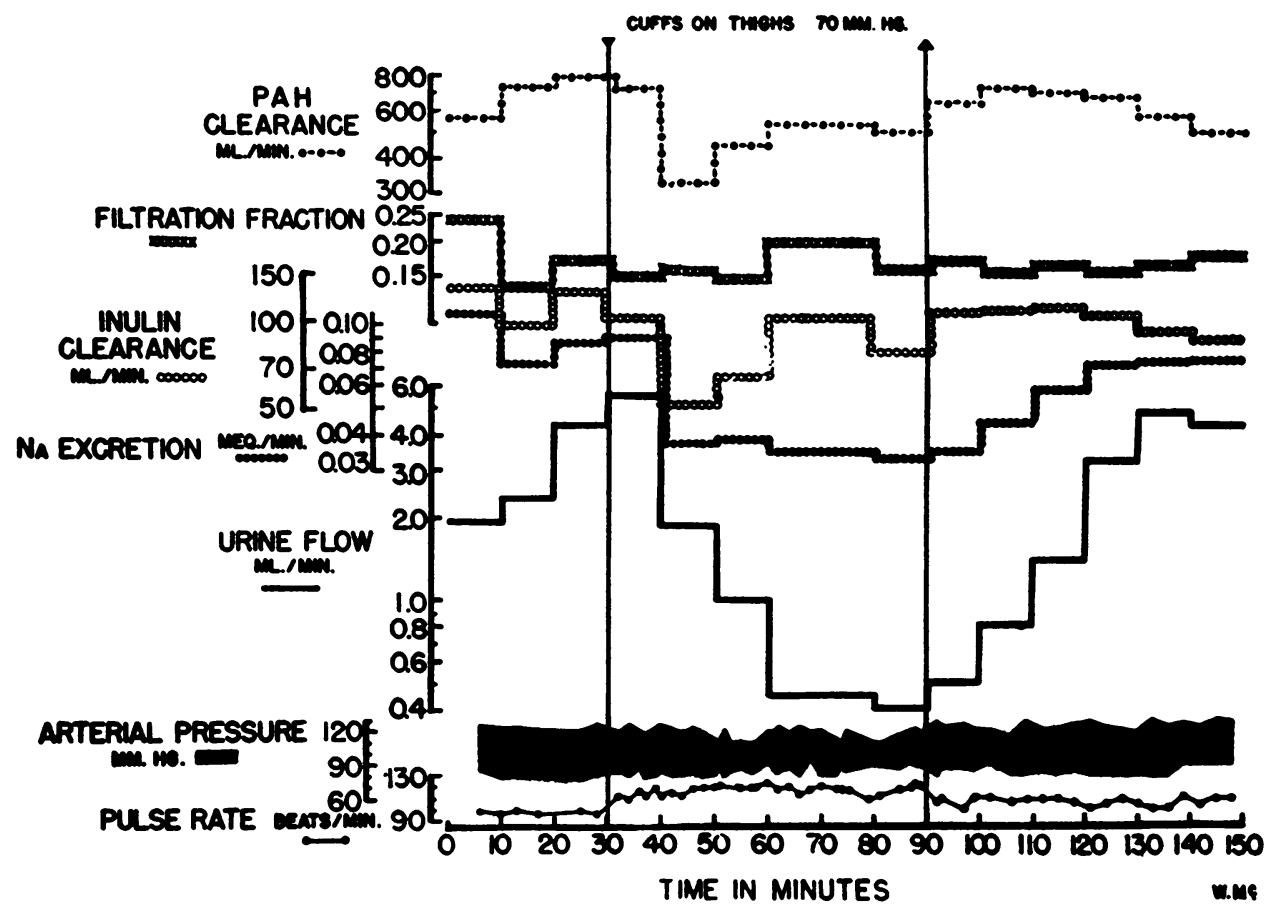

Fig. 1. Chart of Pah Clearance, Filtration Fraction, Inulin Clearance, Sodium Excretion, Urine flow, Arterial Pressure and Pulse Rate in an Experiment on a Normotensive Subject Whose Limbs Were Congested by Inflating Cuffs on the Thighs FOR 60 MinUtes

The ordinate scales are logarithmic except those for arterial pressure and pulse rate.

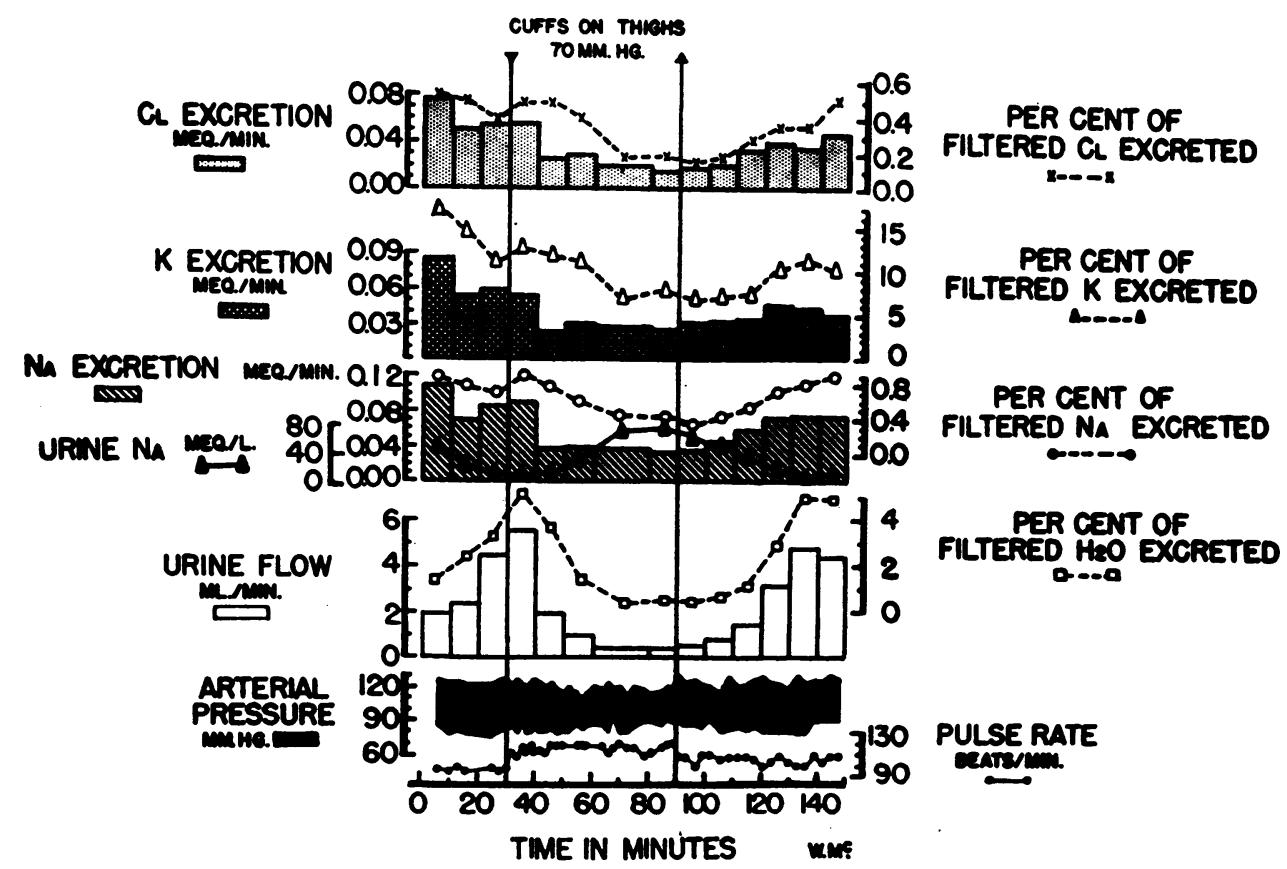

Fig. 2. Chart of the Chloride, Potassium and Sodium Excretions, Urine Sodium Concentration, Urine Flow, and Calculated Per Cent of Filtered Substances Excreted (E/F) in the Experiment Shown in Figure 1

The ordinate scales are all arithmetic. 
of blood were taken $21 / 2$ minutes later. After a good ( 3 to $10 \mathrm{cc}$. per min.) and fairly steady urine flow was established, and when at least three control renal clearance periods had elapsed, the cuffs on the thighs were inflated at slightly less than diastolic pressure (usually at $70 \mathrm{~mm}$. $\mathrm{Hg}$ ). Observations were made for one to six periods, whereupon the congesting cuffs were released and recovery observations were made.

In some experiments during the control period before, and the recovery period after, venous congestion of the limbs, the thigh cuffs were inflated every 5 minutes at the same pressure as used during the congestion period, but only for 30 seconds. This was to accustom the patient to the sensory stimulus of the inflated cuff. In other experiments, after a suitable control period the entire limbs up to the thigh cuffs were compressed by means of a series of cuffs or inflatable leggings (of a "G"-suit) for the same length of time as they were subsequently (after another control period) congested by inflation of the uppermost cuff alone. This compression was designed to cause an equal or greater degree of ischemia, anoxia, and discomfort in the limbs without venous congestion or edema. A number of such additional experiments were done in order to test the effects of other variations in the standard procedures just described upon urine flow, electrolyte ex- cretion, arterial pressure, and pulse rate. These will not be described in detail, but will be mentioned under "Special Results."

Samples of urine and blood were analyzed for clearance substances according to the methods of Goldring and Chasis (4). They were analyzed for sodium and potassium by means of an internal standard flame photometer ${ }^{9}$ and for chloride by the method of Wilson and Ball (5).

Hematocrits of the blood samples were measured in Wintrobe tubes, and the protein content of the plasma by a specific gravity technique (Copper Sulfate).

\section{RESULTS}

\section{General}

Detailed results on a number of patients are shown in the Figures and in Table I, and a statistical analysis of these results is given in Table II. In the experiments shown in Table $I$ the various periods have been grouped and averaged in an attempt to save space without altering significantly

๑ Perkin-Elmer Corporation, Main Avenue, Norwalk, Connecticut.

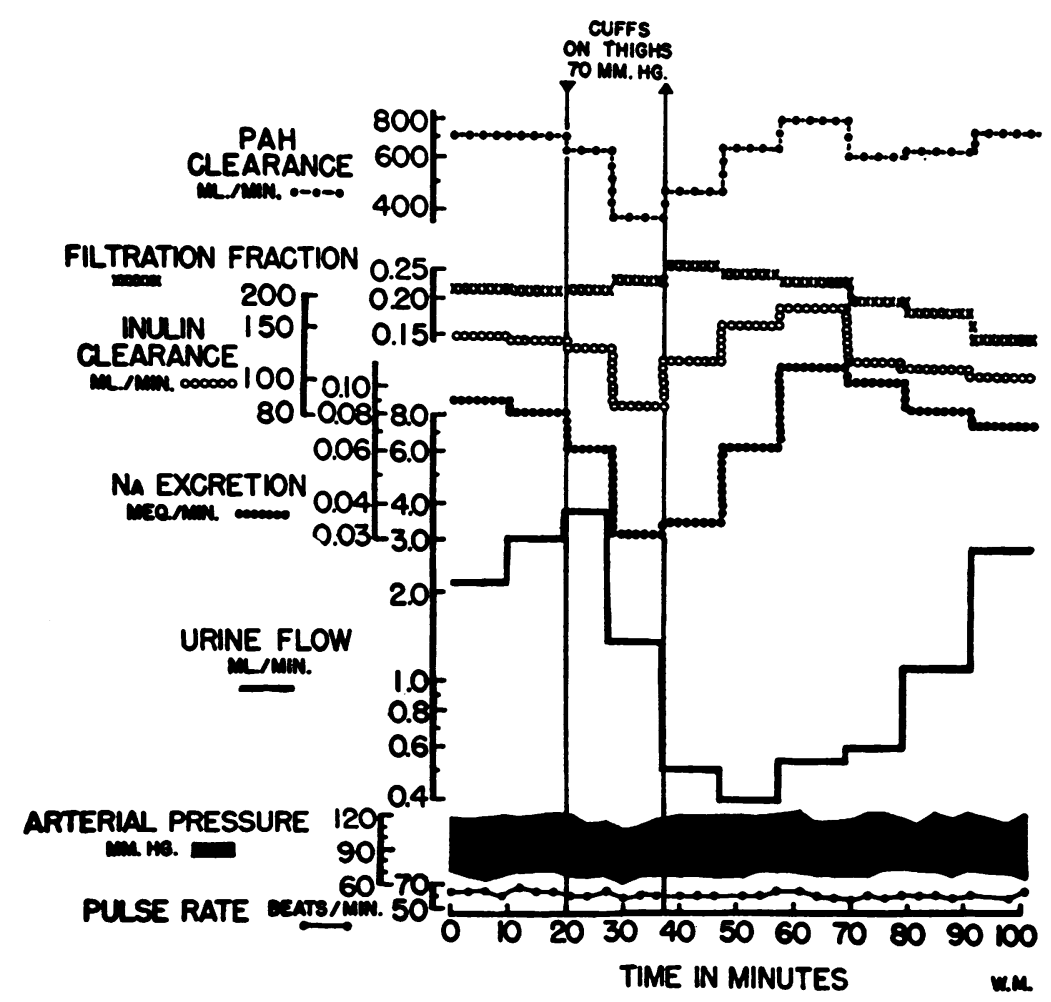

Fig. 3. Chart of an Experiment on a Normotensive Subject Conducted Similarly to That Shown in Figure 1 Except That the Limbs Were Congested for a Shorter (18 Minutes) Period

Method of charting as in Figure 1. 


\begin{tabular}{|c|c|c|c|c|c|c|c|}
\hline \multirow{6}{*}{ 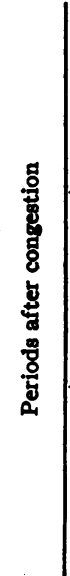 } & \multirow{2}{*}{ 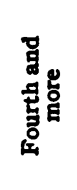 } & $\mathbf{z}$ & DONNNNAN & 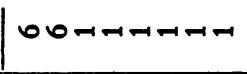 & 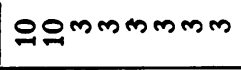 & 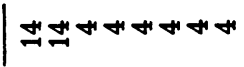 & MINNNNNNA \\
\hline & & $\overbrace{\Sigma}^{5}$ & సิำ & సิ & 落 & 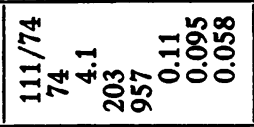 & 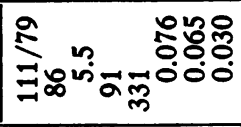 \\
\hline & \multirow{2}{*}{ 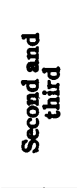 } & $z$ & DONNNNNN & +n- & Nanen & ルேルーナー & Lsenco \\
\hline & & ङ્ّ & 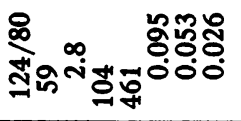 & 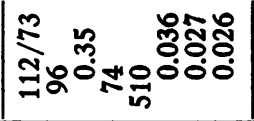 & 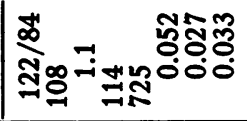 & 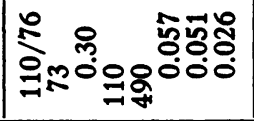 & | \\
\hline & \multirow[b]{2}{*}{ 垔 } & $z$ & ルルナルの & +1 & A-1 & |こニルのルのール & $\sin$ \\
\hline & & ङ & 点 & 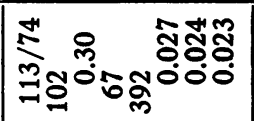 & 我 & 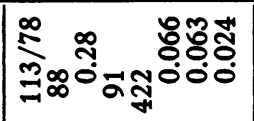 & 悉 \\
\hline \multirow{6}{*}{ 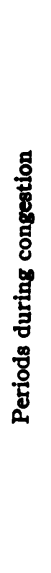 } & \multirow{2}{*}{ 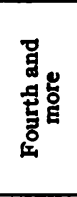 } & $z$ & פONNNNNN & 으음 & M\#NNNNNN & aन्त̄mmmon & 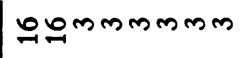 \\
\hline & & 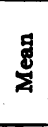 & 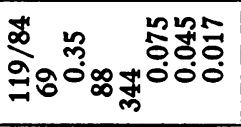 & 尽 & 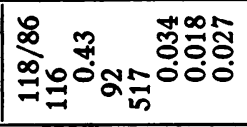 & 蛋 & 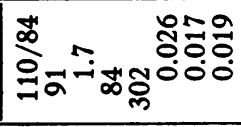 \\
\hline & \multirow{2}{*}{ 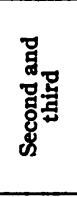 } & z & ตณกNNNNN & INAN & NNNN & 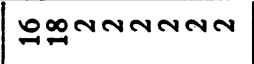 & ザザNNN \\
\hline & & $\sum_{\Sigma}^{5}$ & 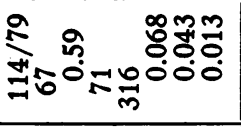 & 츙요 & 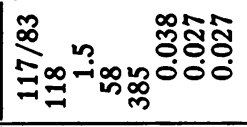 & 尽 & 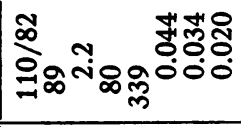 \\
\hline & \multirow[b]{2}{*}{ 蒂 } & z & 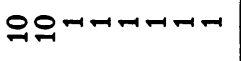 & サનनન & 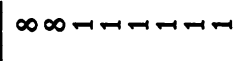 & 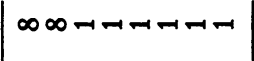 & 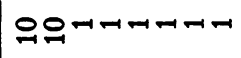 \\
\hline & & ङ્ّ & 令 & 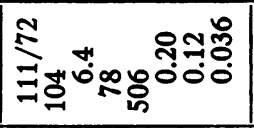 & 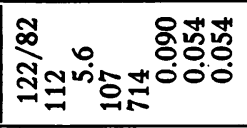 & 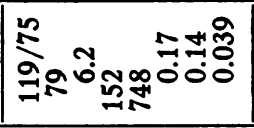 & 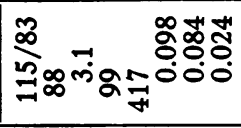 \\
\hline \multirow{4}{*}{ 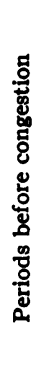 } & \multirow{2}{*}{ 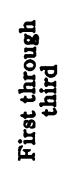 } & z & 음ппㅇ & 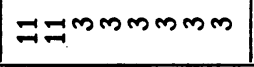 & nलmm & 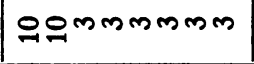 & manm \\
\hline & & ङ્ّ & 点 & 疍 & 离 & 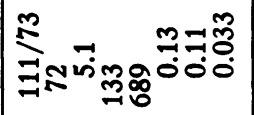 & 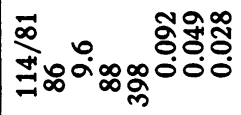 \\
\hline & \multirow{2}{*}{ 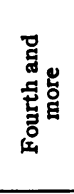 } & $z$ & & 909m & & & \\
\hline & & 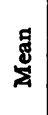 & & 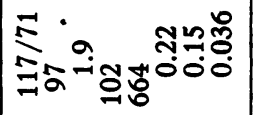 & & & \\
\hline \multirow{2}{*}{\multicolumn{2}{|c|}{ 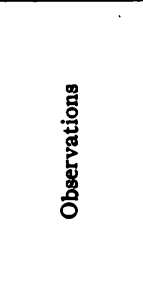 }} & 莣 & 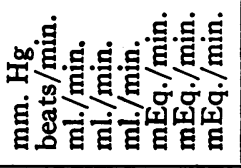 & 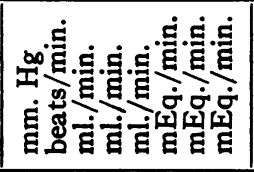 & 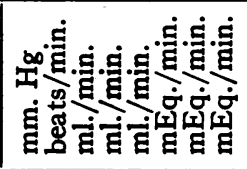 & 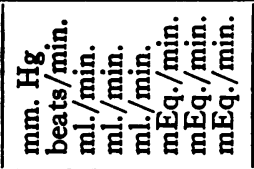 & 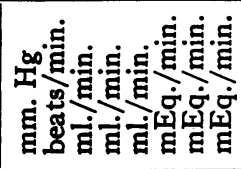 \\
\hline & & 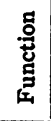 & 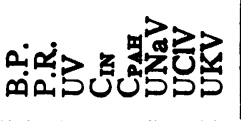 & 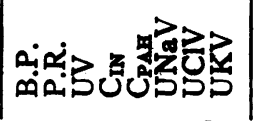 & 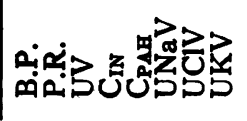 & ma: & 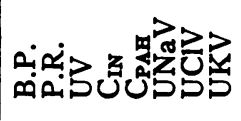 \\
\hline \multicolumn{3}{|c|}{ 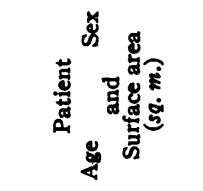 } & $\vec{D}_{\ddot{m}}^{\dot{5}}$ & 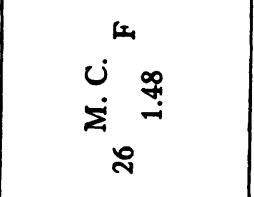 & $\sum_{\sum_{\infty}^{\infty}}^{\Sigma}$ & 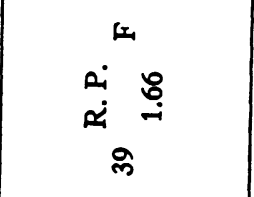 & 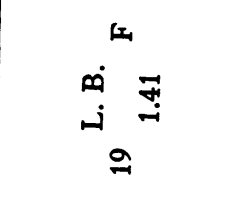 \\
\hline
\end{tabular}




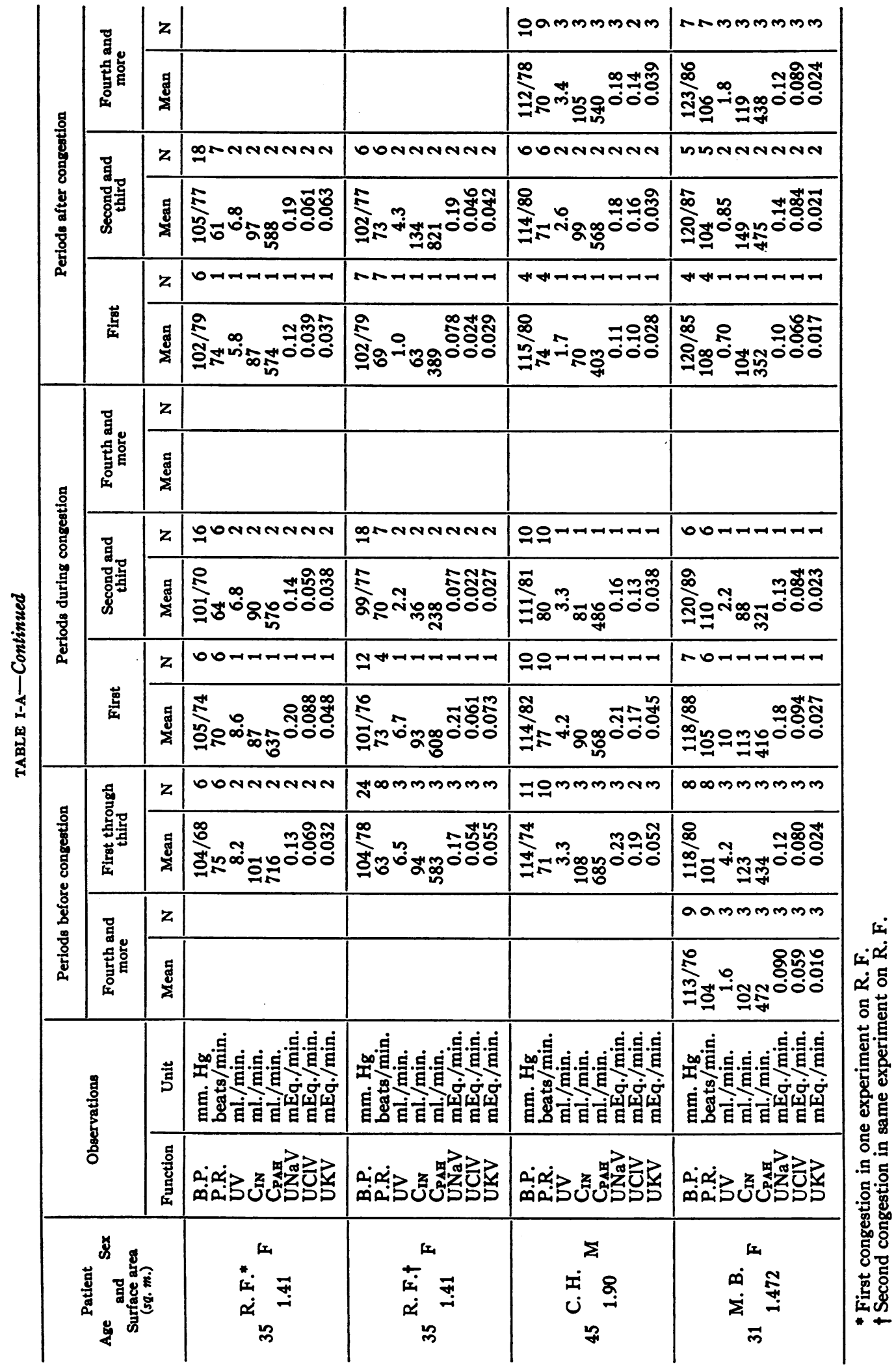




\begin{tabular}{|c|c|c|c|c|c|c|}
\hline \multirow{6}{*}{ 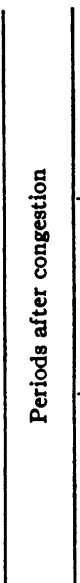 } & \multirow{2}{*}{ 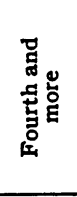 } & z & 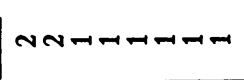 & 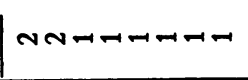 & 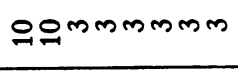 & సָก \\
\hline & & $\stackrel{5}{\Sigma}$ & 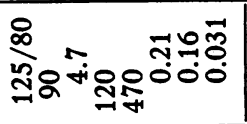 & 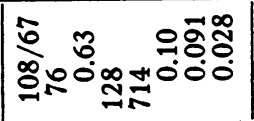 & 尽 n & 旤 \\
\hline & \multirow{2}{*}{ 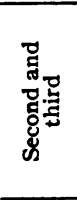 } & $z$ & DONNNNNN & mannNenN & OONNNNNN & ZニNNNNNN \\
\hline & & $\overbrace{\Sigma}^{\Sigma}$ & 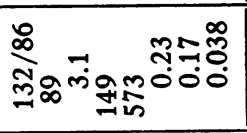 & 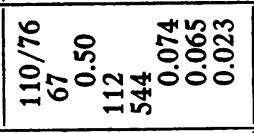 & 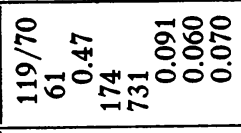 & 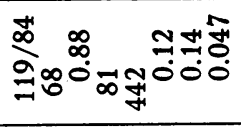 \\
\hline & \multirow[b]{2}{*}{ 营 } & $\mathrm{z}$ & mッールールー & 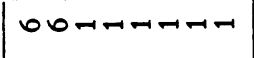 & 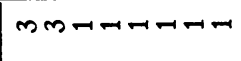 & $\infty$ \\
\hline & & & 勿 & 帘 & 战 & 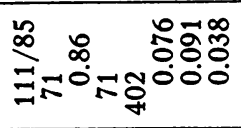 \\
\hline \multirow{6}{*}{ 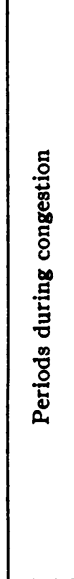 } & \multirow{2}{*}{ 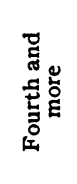 } & $\mathrm{z}$ & & & & \\
\hline & & 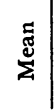 & & & & \\
\hline & \multirow{2}{*}{ 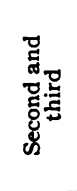 } & $\mathrm{z}$ & ヘヘールのーのー & mッールールール & mmat & \\
\hline & & $\stackrel{\Xi}{\Sigma}$ & 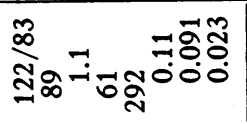 & 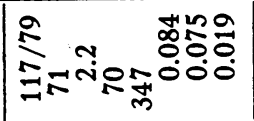 & 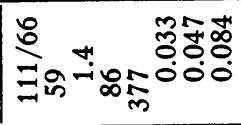 & \\
\hline & \multirow[b]{2}{*}{ 总 } & $\mathrm{z}$ & 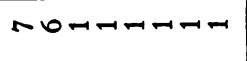 & 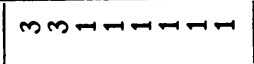 & 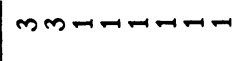 & ヘヘナのナのの゙ \\
\hline & & & 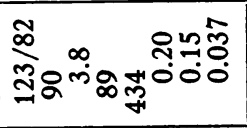 & 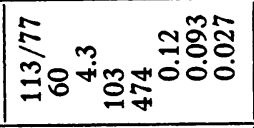 & 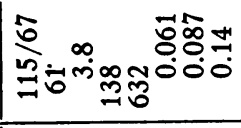 & 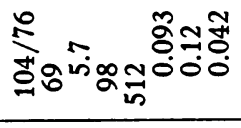 \\
\hline \multirow{4}{*}{ 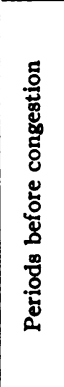 } & \multirow{2}{*}{ 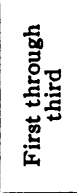 } & $\mathrm{z}$ & 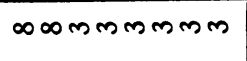 & ZZNNNNNN & mannmana & DONNNNNN \\
\hline & & & 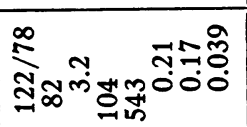 & ô & 余 & 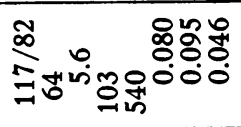 \\
\hline & \multirow{2}{*}{ 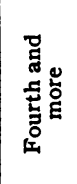 } & $z$ & สบก & & & \\
\hline & & $\overbrace{\Sigma}^{\square}$ & 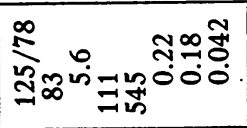 & & & \\
\hline \multirow{2}{*}{\multicolumn{2}{|c|}{ 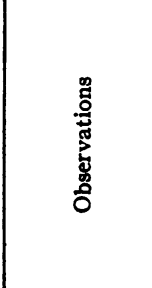 }} & 菅 & 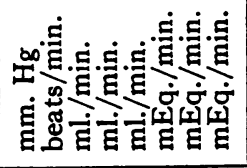 & 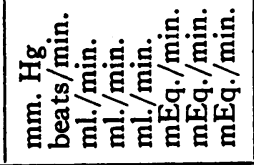 & 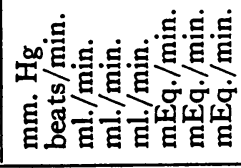 & 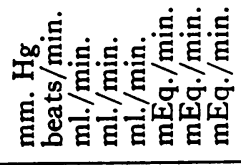 \\
\hline & & 总 & 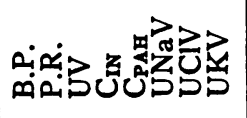 & 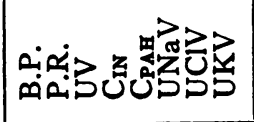 & 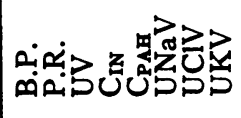 & 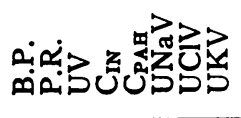 \\
\hline \multicolumn{3}{|c|}{ 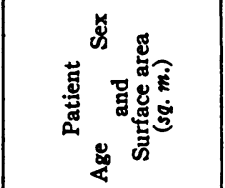 } & 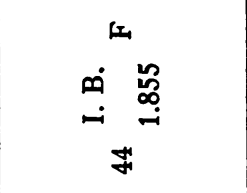 & 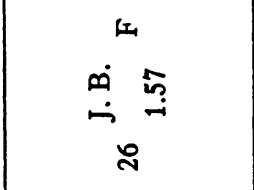 & $\dot{\Sigma}_{i}^{\sum}$ & 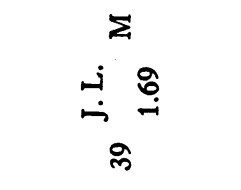 \\
\hline
\end{tabular}




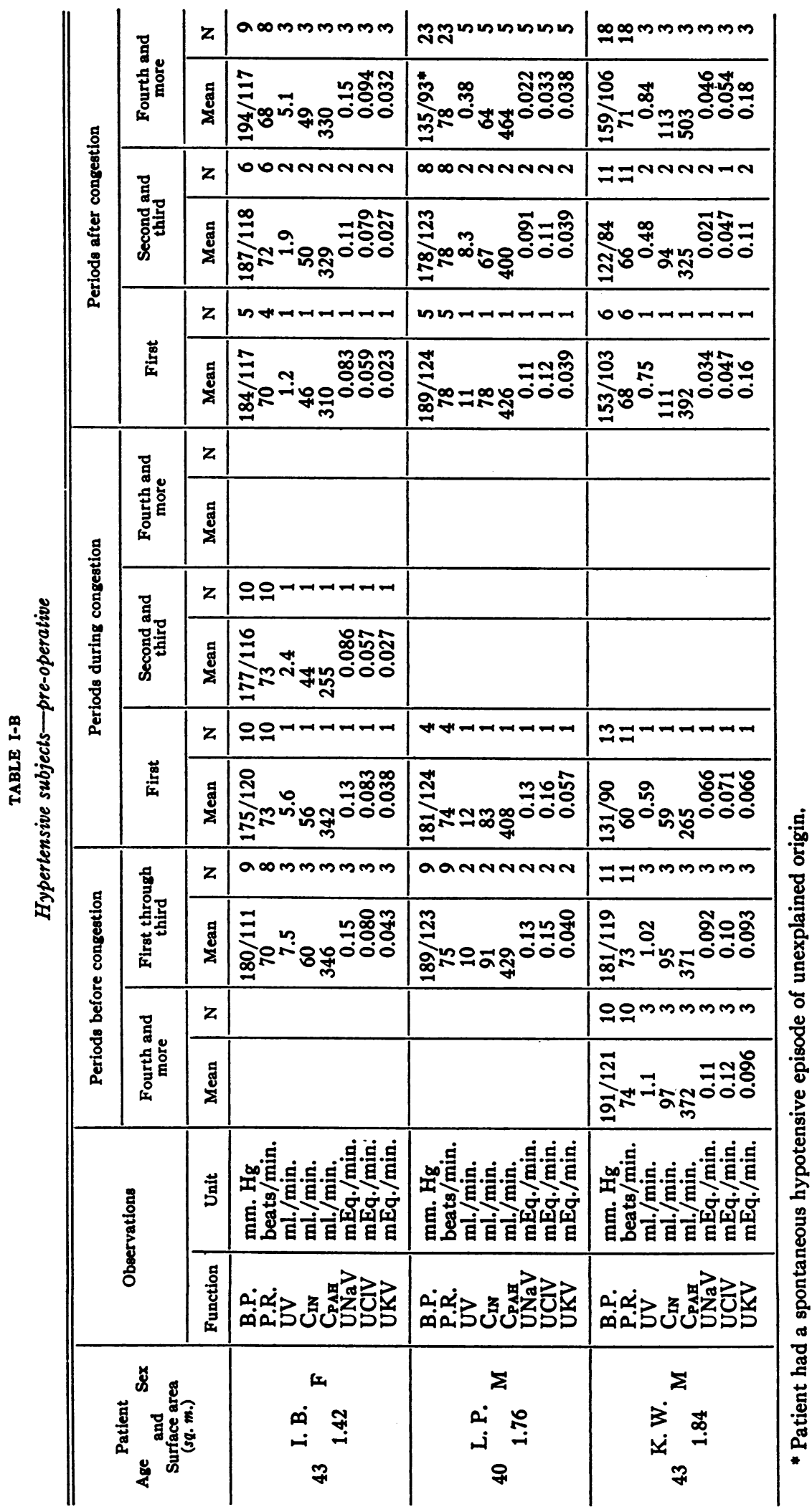




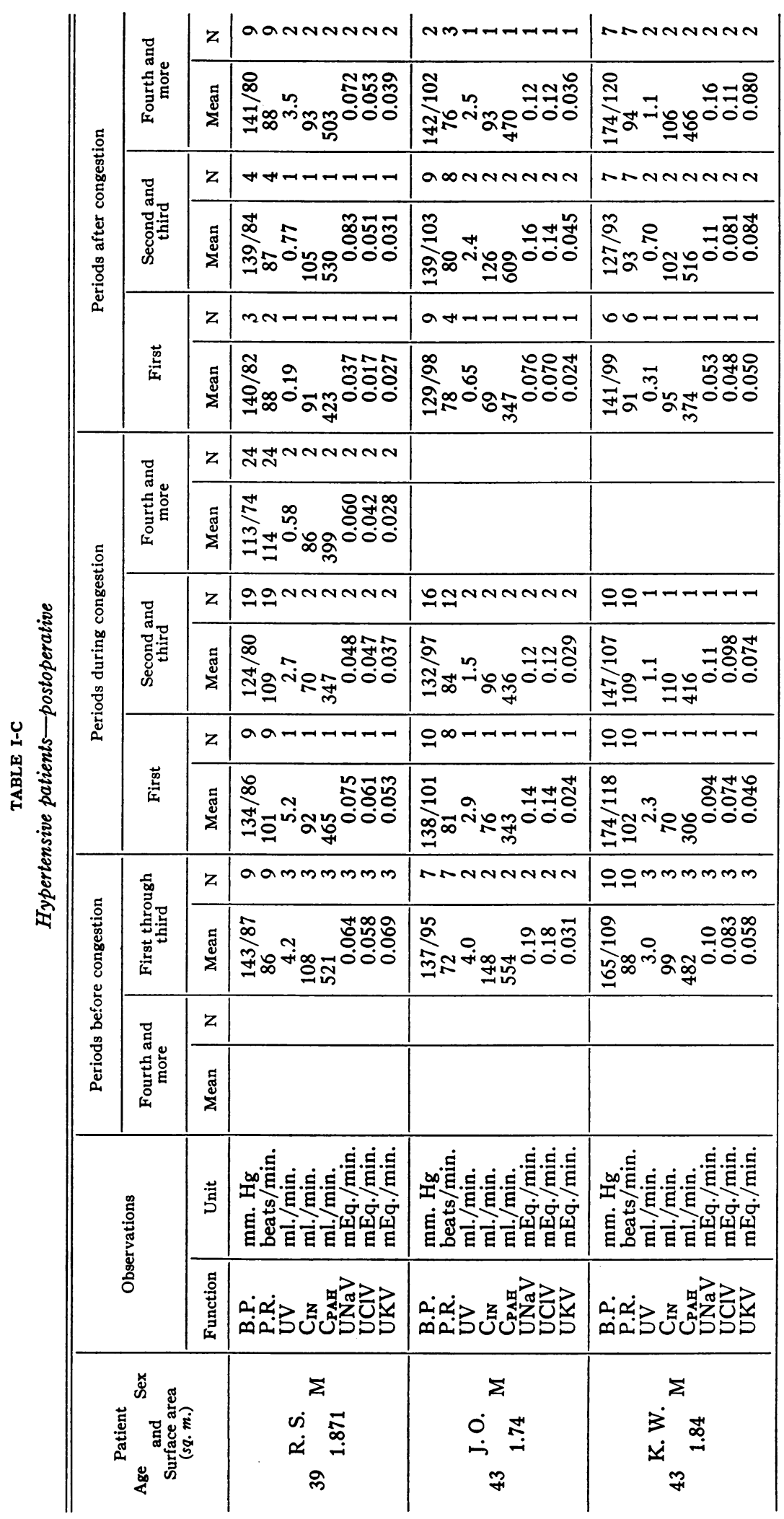




\begin{tabular}{|c|c|c|c|c|c|}
\hline \multirow{6}{*}{ 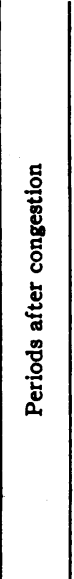 } & \multirow{2}{*}{ 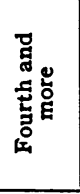 } & $z$ & mannment & DOOODODNO & DopNANAN \\
\hline & & $\stackrel{\Xi}{\Sigma}$ & 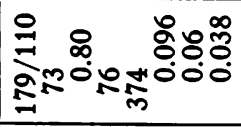 & 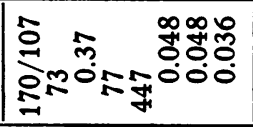 & 年 \\
\hline & \multirow{2}{*}{ 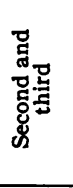 } & $z$ & MISNNNNNA & mannenen & MANNNNAN \\
\hline & & $\sum_{\Sigma}^{5}$ & 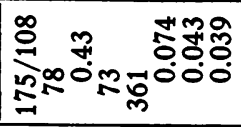 & 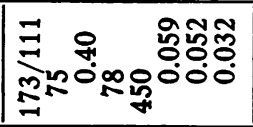 & 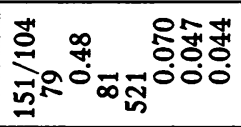 \\
\hline & \multirow[b]{2}{*}{ 蒂 } & $z$ & 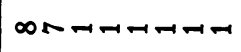 & ๓ッカーカーカー & のロールールール \\
\hline & & $\mathbb{g}_{\Sigma}^{5}$ & 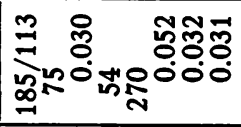 & 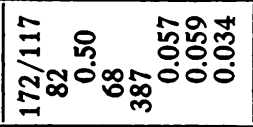 & స్త్రి \\
\hline \multirow{6}{*}{ 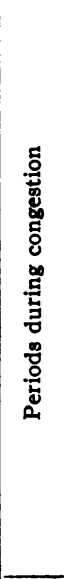 } & \multirow{2}{*}{ 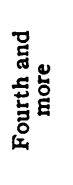 } & $z$ & & & \\
\hline & & $\stackrel{\Xi}{\Xi}$ & & & \\
\hline & \multirow{2}{*}{ 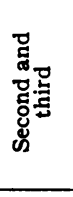 } & $z$ & 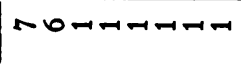 & 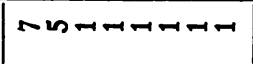 & \\
\hline & & Ӗ్ّ & 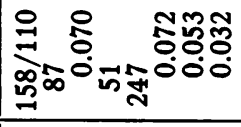 & 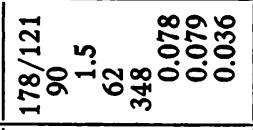 & \\
\hline & \multirow[b]{2}{*}{ 蒂 } & z & ดのルールールー & | & エエルのルールー \\
\hline & & $\stackrel{5}{\Sigma}$ & 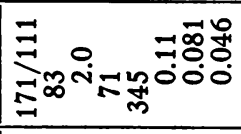 & 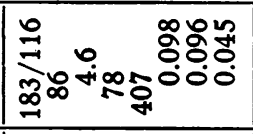 & 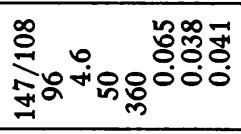 \\
\hline \multirow{4}{*}{ 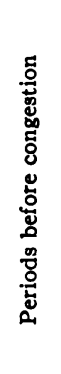 } & \multirow{2}{*}{ 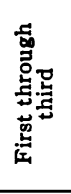 } & z & 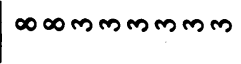 & MINNNNNNA & $\Rightarrow=$ момпмо \\
\hline & & $\sum_{\Sigma}^{5}$ & 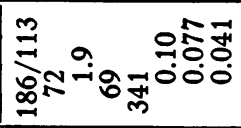 & 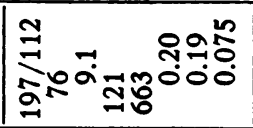 & 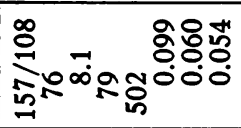 \\
\hline & \multirow{2}{*}{ 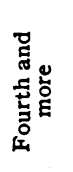 } & $z$ & & & \\
\hline & & $\stackrel{\Xi}{\Xi ّ}$ & & & \\
\hline \multirow{2}{*}{\multicolumn{2}{|c|}{ 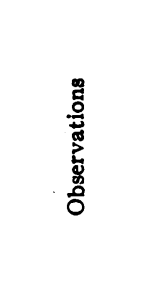 }} & 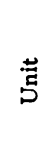 & 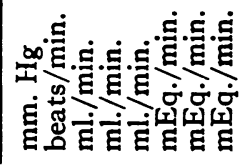 & 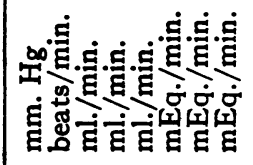 & 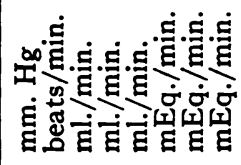 \\
\hline & & 䒿 & 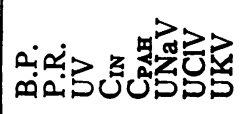 & 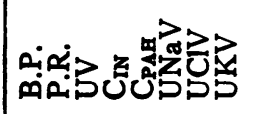 & 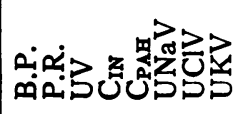 \\
\hline \multicolumn{3}{|c|}{ 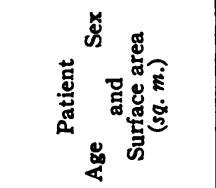 } & 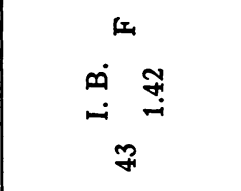 & 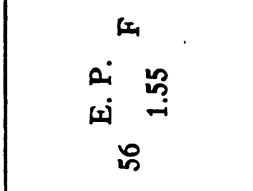 & 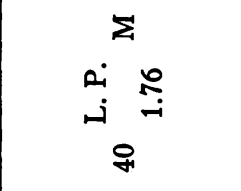 \\
\hline
\end{tabular}


the true trends of the results. ${ }^{10}$ It will be seen that in general the effects of venous congestion in the limbs upon renal clearances and the excretion of water and salt were essentially similar in the normotensive control subjects (Table I-A and Figures 1-4) and in the hypertensive patients both before (Table I-B) and after splanchnicectomy (Table I-C and Figures 5,6). Thus, characteristically after about ten minutes of limb congestion, the urine flow began to decrease, frequently falling to onetenth its previous rate. With the initial fall in

10 Thus the control period immediately before congestion of the limbs (first period before) has been averaged together with the two prior periods under "First through third." When more than three control periods were used all the earlier periods have been averaged under "Fourth and more." The periods during congestion and after its release have also been averaged together in a similar way, except for the first period in each case, which has been recorded separately as probably not indicative of the subsequent general trend. Likewise, in the statistical analysis in Table II comparisons have been made only between those portions of the experiments which are representative of a relatively "steady state." urine flow there was a decrease in calculated renal plasma flow (PAH clearance) and filtration rate (inulin clearance) and in observed sodium excretion, chloride excretion, and sometimes potassium excretion. The percentage decrease in excretion of electrolytes and clearance substances was not so great as of water, so that the concentration of these materials in the urine usually rose, often to a marked degree (Figures 2, 4, 6).

Not only was the reduction of urine flow greater in amount, but it was also usually longer in duration than the other renal changes produced by venous congestion of the limbs. Thus, during the first part of the long (40- to 60-minute) congestion experiments (Figures 1, 2, 5, 6) the urine flow decreased, and it usually remained at the lower levels, even during the last 20 to 30 minutes of congestion when the calculated renal plasma flow and glomerular filtration rate (and occasionally the electrolyte excretion) were often returning towards if not to control levels. Sodium, chloride, and potassium excretion usually remained definitely below control

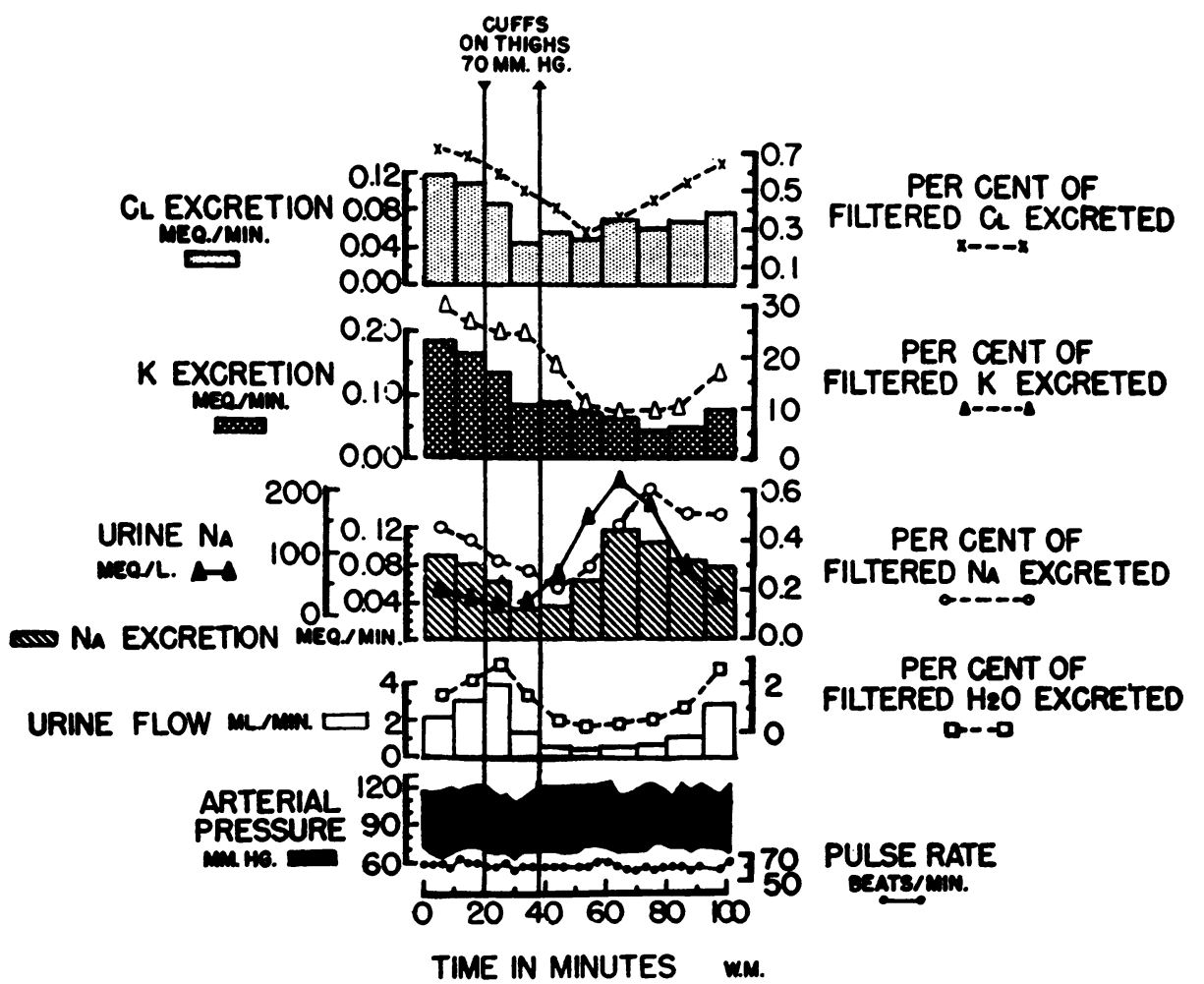

Fig. 4. Chart of Electrolyte and Water Excretion during the Experiment Shown IN FIgURE 3

Method of charting as in Figure 2. 


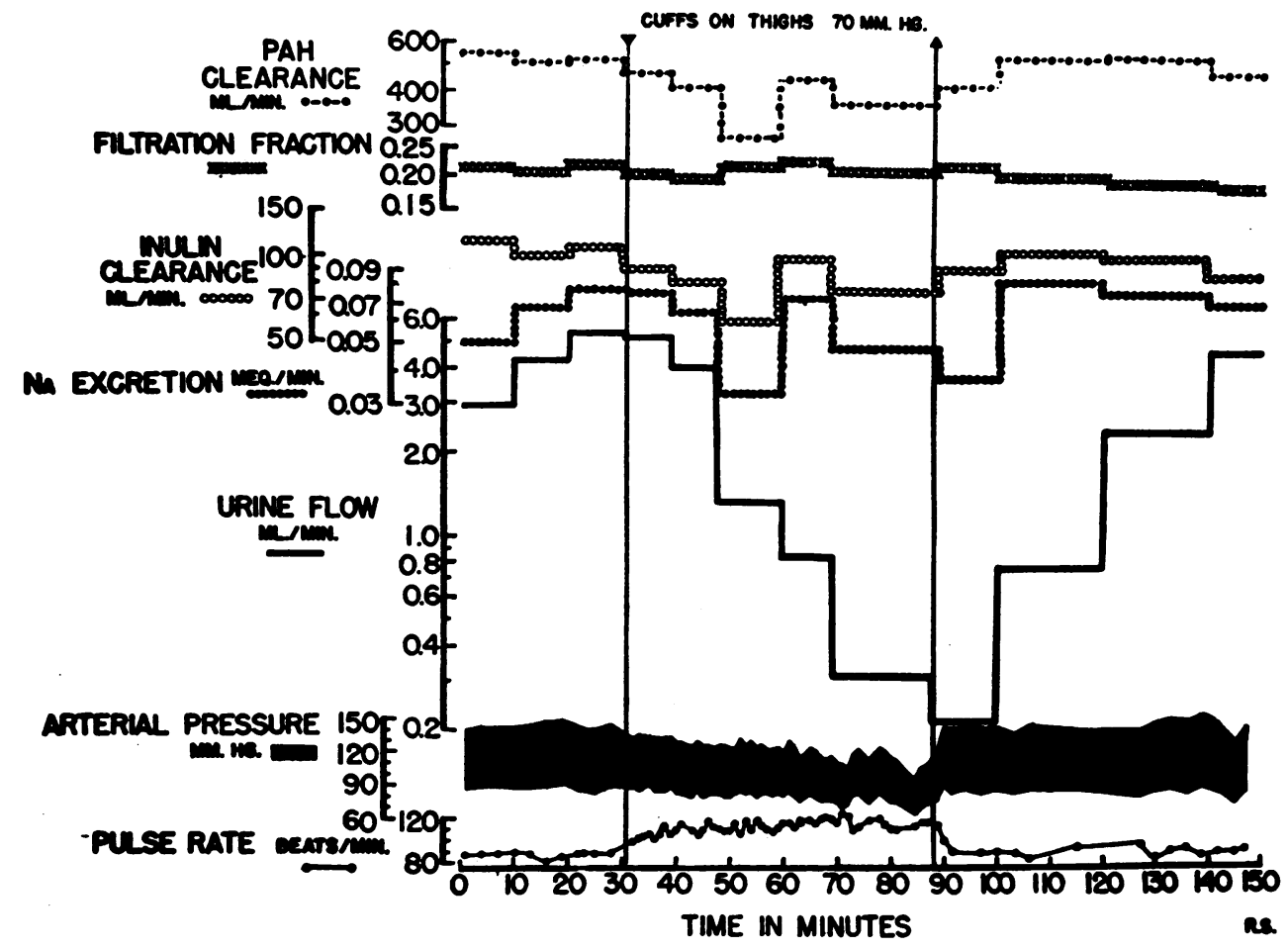

Fig. 5. Chart of an Experiment on a Hypertensive Subject Two Weeks After a Lumbodorsal Sympathectomy Conducted Similarly to that Shown in Figure 1

Method of charting as in Figure 1.

levels throughout the congestion period. On release of the congesting cuffs there was often a further slight to moderate decrease in urine volume and electrolyte excretion for one or two periods, then a gradual return to control levels, the urine flow usually returning more slowly than the electrolyte excretion.

During short (10- to 30 -minute) periods of venous congestion similar effects occurred (Figures 3 and 4 ), although usually not to the same degree as during the longer periods, so that by the time the congesting cuffs were released the urine flow and other renal functions, though decreased, had usually not fallen to their minimum values. After release of the cuffs these functions continued to fall for one or two periods; indeed, it appeared in many of the shorter tests as if the release of the congesting cuffs was a brief stimulus to further small reductions in urine flow, renal clearances, and sodium excretion. Even including these reductions, the total decreases in sodium excretion as a rule were not as great as from the long periods of congestion, although the decreases in urine flow were about as great. After release of the cuffs the urine flow often returned more slowly than the other functions, whereas renal plasma flow, glomerular filtration rate and sodium and chloride excretion tended to increase promptly together. Potassium excretion behaved more irregularly, but usually fell during congestion and returned to control levels after release but not necessarily simultaneously with sodium and chloride excretion.

\section{General Hemodynamic Effects}

As a rule pulse rate increased moderately during the period of venous congestion, while systolic and diastolic arterial pressures were not greatly changed. Sometimes, however, pulse rate rose markedly and occasionally arterial pressure fell (Figure 5), rarely even to collapse levels. When a definite hypotensive reaction occurred it was always followed by very marked responses of the same general type as already described, the antidiuresis being particularly profound and sometimes persisting for more than an hour after release of 
TABLE II

Statistical analysis

\begin{tabular}{|c|c|c|c|c|c|c|c|c|c|c|}
\hline & & $\begin{array}{l}\text { Systolic } \\
\text { pressure } \\
\text { mm. } \mathrm{Hg}_{\mathrm{g}}\end{array}$ & $\begin{array}{c}\text { Diastolic } \\
\text { pressure } \\
m m . H_{g}\end{array}$ & $\begin{array}{l}\text { Pulse } \\
\text { rate } \\
\text { beats/ } \\
\text { min. }\end{array}$ & $\begin{array}{l}\text { UV } \\
\text { ml. } / \text { min. }\end{array}$ & $\underset{m l . / m i n}{\text { CIN }}$ & $\underset{\text { ml. }}{\text { CPAB }}$ & $\begin{array}{c}\mathrm{UNaV} \\
\text { mEq./ } \\
\text { min. }\end{array}$ & $\begin{array}{c}\text { UClV } \\
\text { mEq./ } \\
\text { min. }\end{array}$ & $\begin{array}{c}\text { UKV } \\
\text { mEq./ } \\
\text { min. }\end{array}$ \\
\hline $\begin{array}{c}\text { Periods before } \\
\text { congestion } \\
\text { First through } \\
\text { third } \\
\text { (Control) } \\
\end{array}$ & $\begin{array}{l}\text { Mean }(n=18) \\
\text { Standard error of mean }\end{array}$ & $\begin{array}{r}132 \\
7\end{array}$ & $\begin{array}{r}85 \\
4\end{array}$ & $\begin{array}{r}77 \\
3\end{array}$ & $\begin{array}{l}5.1 \\
0.6\end{array}$ & $\begin{array}{r}107 \\
6\end{array}$ & $\begin{array}{r}554 \\
30\end{array}$ & $\begin{array}{l}0.14 \\
0.013\end{array}$ & $\begin{array}{l}0.10 \\
0.012\end{array}$ & $\begin{array}{l}0.051 \\
0.008\end{array}$ \\
\hline $\begin{array}{l}\text { Periods during } \\
\text { congestion } \\
\text { Second and } \\
\text { third }\end{array}$ & $\begin{array}{l}\text { Mean }(n=18) \\
\text { Standard error of mean } \\
\text { Mean difference from control } \\
\text { Standard error of difference } \\
\text { Significance of difference }(P)\end{array}$ & $\begin{array}{l}126 \\
6 \\
-6 \\
2 \\
0.03\end{array}$ & $\begin{array}{r}87 \\
4 \\
3 \\
1 \\
0.02\end{array}$ & $\begin{array}{c}87 \\
4 \\
10 \\
2 \\
<0.01\end{array}$ & $\begin{array}{c}2.0 \\
0.3 \\
-3.0 \\
0.6 \\
<0.01\end{array}$ & $\begin{array}{r}71 \\
5 \\
-36 \\
5 \\
<0.01\end{array}$ & $\begin{array}{r}360 \\
22 \\
-194 \\
26 \\
<0.01 \\
\end{array}$ & $\begin{array}{r}0.088 \\
0.008 \\
-0.050 \\
0.007 \\
<0.01\end{array}$ & $\begin{array}{r}0.067 \\
0.007 \\
-0.036 \\
0.008 \\
<0.01 \\
\end{array}$ & $\begin{array}{r}0.032 \\
0.004 \\
-0.018 \\
0.005 \\
<0.01 \\
\end{array}$ \\
\hline Fourth and more & $\begin{array}{l}\text { Mean }(n=6) \\
\text { Standard error of mean } \\
\text { Mean difference from control } \\
(n=6) \\
\text { Standard error of difference } \\
\text { Significance of difference (P) }\end{array}$ & $\begin{array}{r}114 \\
2 \\
-5 \\
\\
\quad 6 \\
0.46\end{array}$ & $\begin{array}{r}81 \\
2 \\
3 \\
3 \\
0.39\end{array}$ & $\begin{array}{r}100 \\
7 \\
18 \\
4 \\
40.01\end{array}$ & $\begin{array}{r}0.6 \\
0.2 \\
-4.9 \\
1.0 \\
<0.01\end{array}$ & $\begin{array}{r}80 \\
5 \\
-24 \\
9 \\
9.05\end{array}$ & $\begin{array}{r}382 \\
30 \\
-179 \\
43 \\
<0.01 \\
\end{array}$ & $\begin{array}{r}0.052 \\
0.008 \\
-0.064 \\
0.029 \\
0.08\end{array}$ & $\begin{array}{r}0.036 \\
0.008 \\
-0.044 \\
0.016 \\
0.04\end{array}$ & $\begin{array}{r}0.022 \\
0.001 \\
-\mathbf{0 . 0 2 2} \\
0.006 \\
0.02\end{array}$ \\
\hline $\begin{array}{l}\text { Periods after } \\
\text { congestion } \\
\text { Fourth and } \\
\text { more }\end{array}$ & $\begin{array}{l}\text { Mean }(n=15) \\
\text { Standard error of mean } \\
\text { Mean difference from congestion } \\
\text { periods second and third }(n=15) \\
\text { Standard error of difference } \\
\text { Significance of difference }(P) \\
\text { Mean diference from congeetion } \\
\text { periods fourth and more }(n=5) \\
\text { Standard error of difference } \\
\text { Significance of difference }(P) \\
\text { Mean difference from control } \\
\text { (n=15) } \\
\text { Standard error of difference } \\
\text { Significance of difference (P) }\end{array}$ & $\begin{array}{l}137 \\
7 \\
8 \\
3 \\
<0.01 \\
9 \\
5 \\
0.14 \\
1 \\
3 \\
0.76\end{array}$ & $\begin{array}{l}89 \\
4 \\
-1 \\
2 \\
0.56 \\
-2 \\
2 \\
0.37 \\
2 \\
1 \\
0.18\end{array}$ & $\begin{array}{l}81 \\
4 \\
-9 \\
2 \\
<0.01 \\
-14 \\
4 \\
0.02 \\
2 \\
1 \\
0.11\end{array}$ & $\begin{array}{l}2.8 \\
0.6 \\
1.1 \\
0.5 \\
0.05 \\
3.2 \\
0.9 \\
0.02 \\
-\mathbf{2 . 0} \\
0.8 \\
\mathbf{0 . 0 3}\end{array}$ & $\begin{array}{c}96 \\
5 \\
25 \\
5 \\
<0.01 \\
9 \\
4 \\
0.08 \\
-11 \\
5 \\
0.06\end{array}$ & $\begin{array}{c}492 \\
30 \\
142 \\
30 \\
<0.01 \\
107 \\
40 \\
0.06 \\
-41 \\
22 \\
0.09\end{array}$ & $\begin{array}{c}0.11 \\
0.012 \\
0.026 \\
0.008 \\
<0.01 \\
0.026 \\
0.008 \\
0.03 \\
-0.028 \\
0.016 \\
0.11\end{array}$ & $\begin{array}{c}0.082 \\
\mathbf{0 . 0 0 9} \\
0.014 \\
0.006 \\
\mathbf{0 . 0 3} \\
\mathbf{0 . 0 2 1} \\
\mathbf{0 . 0 0 7} \\
\mathbf{0 . 0 4} \\
\mathbf{0 . 0 2 6} \\
\mathbf{0 . 0 1 2} \\
\mathbf{0 . 0 5}\end{array}$ & $\begin{array}{c}0.038 \\
0.004 \\
0.005 \\
0.002 \\
0.08 \\
0.011 \\
0.002 \\
<0.01 \\
-0.015 \\
0.008 \\
0.08\end{array}$ \\
\hline
\end{tabular}

the cuffs, whereas the arterial pressure usually returned promptly to control levels.

Hypertensive patients, though usually more unstable in their blood pressure reactions, showed no striking differences after as compared with before lumbodorsal splanchnicectomy, or as compared with the more normal control subjects. During venous congestion of the limbs the changes in arterial pressure, pulse rate, urine flow and renal clearances were generally similar to those already described in the normal subjects.

\section{SPECIAL RESULTS}

\section{Hematologic Effects}

During and after venous congestion of the limbs there was, if anything, a tendency for the arterial blood hematocrit and protein content to rise, particularly immediately following the release of the congesting cuffs.

\section{Effects of Sensory Stimulation and Discomfort}

Because pain and even moderate discomfort are known to be potent antidiuretic stimuli in animals, care was taken to avoid such factors as much as possible in the course of the tests described above.
In addition, some experiments were done deliberately (as mentioned under "Methods") to determine the role of these factors. Thus, it was found that inflation of the congesting cuffs for only 30 seconds at five-minute intervals produced no appreciable effects upon the hemodynamic or renal functions measured. Furthermore, continuous compression of the entire limbs of a similar degree and duration as the congestion used at a different time caused no consistent effects on the measurements made. There was often a moderate increase in diuresis during such compression, although occasionally there was a brief, slight decrease. However, patients uniformly reported this procedure to be distinctly more uncomfortable than congestion of the limbs at the same pressure.

\section{Effects of Emotion}

Occasionally, particularly at the end of long experiments, patients became fatigued and emotionally upset. It was interesting, in view of the possible influences of this reaction upon renal and circulatory measurements being made, that it had no consistent effects. Indeed, when venous congestion was applied during such a reaction, patients as often as not failed to have the antidiuresis and 
associated renal changes that they characteristically had when under no discernible emotional stress. On the other hand, occasional patients (e.g., patient R. F.) failed to show the characteristic reactions early in a test, although they did later. In these cases it usually appeared that there had been either an inadequate rest period for hemodynamic and body fluid adjustments to the experimental condition (resting horizontal position) to come into equilibrium; or a preliminary over-hydration with ingestion of a large amount of fluid prior to the test.

\section{Effects of Venous Congestion of Other Areas or at Other Pressures}

A number of incomplete "pilot" experiments were done in which the arms were congested instead of the legs or in which either area was congested at 30 instead of $70 \mathrm{~mm}$. $\mathrm{Hg}$. In general, the effects of congesting the arms or of using lower congesting pressures on the thighs upon water and electrolyte excretion were the same qualitatively though smaller quantitatively, as compared with the effects of congesting the legs at higher pressures.

\section{Effects of Urine Volume}

Early in this work it became apparent that the most marked if not the most consistent renal change produced by venous congestion of limbs in a patient under diuresis was antidiuresis. Indeed, it was considered possible that this was the only primary renal change effected by venous congestion and that other apparent changes were either secondary to this or were even artefacts due to the use of methods dependent upon adequate urine flow. Attempts were made to rule out these possibilities by careful recalculation of the data so as to compare the average clearances per minute for all the periods combined during the entire antidiuretic response (from full diuresis back to full diuresis), with the average of the combined control values before and after the antidiuresis. The average clearances for these combined periods in nearly every case clearly indicated reductions of renal plasma flow and glomerular filtration rate

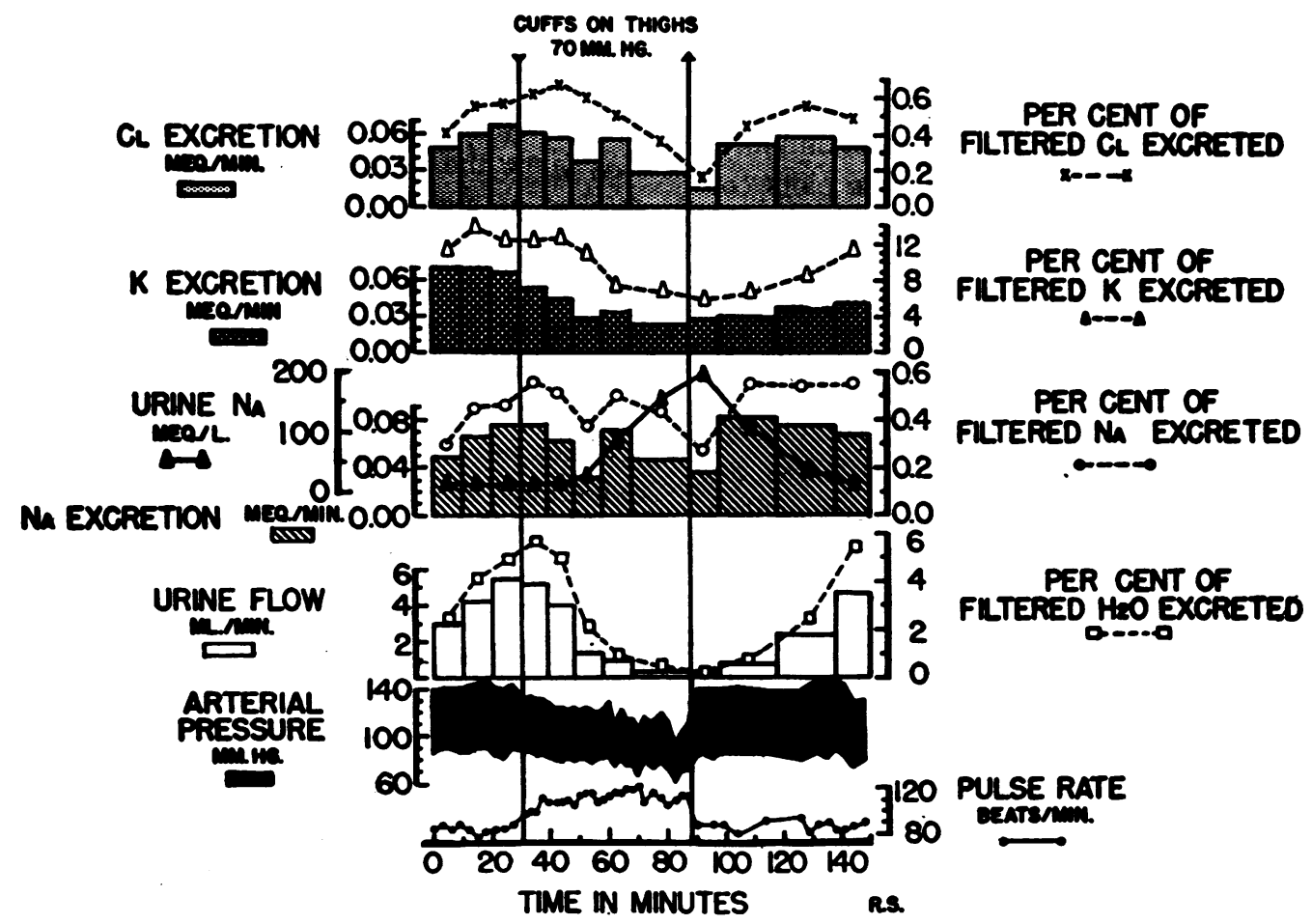

Fig. 6. Chart of Electrolyte and Water Excretion during the Experiment Shown in Figure 5 Method of charting as in Figure 2. 
during the antidiuretic response, but these were of a degree ordinarily considered insignificant for single short periods. It was therefore judged impossible to decide conclusively by this method how greatly "dead space" and "washout" errors contributed to the apparent results, although in certain patients in whom obvious overshoots in clearance occurred on reappearance of diuresis (e.g., R. P., R. F., L. B., W. M., G. V., R. S., and J. O.) this seemed quite substantial. In other patients, however, the changes in clearances and electrolyte excretion bore no (or even an opposite) relationship to urine flow. For example, in patient M. C. urine flow remained low after venous congestion was released whereas the clearance of inulin and $\mathrm{PAH}$ returned to control values. The same was true in patient J. B., although in this patient sodium excretion also returned to control values, whereas in M. C. it remained low with the urine flow. Further, sodium excretion in occasional patients (G. V. and J. L.) failed to be lowered significantly, although UV, $\mathrm{C}_{\text {IN }}$ and $\mathrm{C}_{\mathbf{P A B}}$ decreased in the usual manner.

Another type of recalculation of the data was made in an attempt to shed light on these points. The average percentage change (from control) in each function (clearance) during each period was calculated for the entire normotensive, and the entire postoperative hypertensive groups, and plotted as two (average) experiments. These plots confirmed the impressions already gained from plots of separate experiments, namely that although initially (in the early congestion periods) all the functions decreased sharply together, later on (4th through 6th periods) the urine flow (water excretion) was decreased most, sodium and chloride less (and about equally), potassium still less, and $\mathrm{PAH}$ (plasma flow) and inulin (glomerular filtration rate) least, but nevertheless were definitely decreased. Furthermore, the responses of the normotensive subjects were not significantly different from those of the postoperative hypertensive patients.

These results suggested that although the urine volume and the clearances of inulin, para-aminohippurate, and sodium usually decreased and increased together during and after venous congestion of the limbs, they did not necessarily do so equally and that although the changes in urine volume might magnify the apparent changes in clear- ances, the latter appeared to be essentially independent effects of the venous congestion and its release. These opinions were confirmed definitely as regards sodium excretion and tentatively as regards renal plasma flow and glomerular filtration rate in subsequent experiments in patients with diabetes insipidus and in normal subjects under mannitol diuresis $(6,7)$. In these experiments there were minimal changes in urine volume during venous congestion of the limbs, although there were the usual changes in sodium excretion and similar (though less marked) changes in clearance of inulin and PAH.

\section{Effect of Changes in Glomerular Filtration Rate (Inulin Clearance)}

Additional evidence that the apparent changes in electrolyte excretion during and after venous congestion were not entirely artefacts due to collection errors but were, in part at least, the result of real differences (whether primary or secondary) in the treatment by the tubules of the filtrate presented to them was shown by calculating the "Tubular rejection fraction" or "Percentage of filtered substance excreted" (E/F) (Figures 2, 4, 6). (Space does not permit inclusion of these data in the tables, but their nature can be determined by calculating the ratio, $\mathrm{UV}$ times $\mathrm{Na}, \mathrm{K}$ or $\mathrm{Cl}$, over $\mathrm{C}_{\mathrm{IN}}$, since the plasma levels of these electrolytes showed no significant changes during the experiments.) The calculations usually showed the percentage of the filtered water, sodium, chloride and, to a lesser degree, potassium which was actually excreted in the urine to be decreased during venous congestion and immediately after its release, and at times not to return to control levels until after reductions in glomerular filtration rate (inulin clearance) had disappeared. However, there was no uniform pattern in the behavior of sodium $\mathrm{E} / \mathrm{F}$. Thus, during and after the shorter congestion experiments the decreases in $\mathrm{E} / \mathrm{F}$ of sodium were generally less than during and after the longer congestion periods, although the other results might be comparable. Therefore, in the shorter experiments it appeared that the excretion of sodium bore a fairly "passive" or proportional relationship to glomerular filtration rate. However, in the longer experiments sodium excretion appeared to take a more independent course, resulting in greater 
changes in E/F. Thus, patients M. C. and R. P. showed further decreases in the excretion and $\mathrm{E} / \mathrm{F}$ of sodium at the end of the congestion period when glomerular filtration rate was increasing, while patient W. Mc. showed an unchanged excretion with a decreased $\mathrm{E} / \mathrm{F}$ at that time. In patient $\mathrm{L}$. B., on the other hand, the changes in glomerular filtration rate were never great, while the typical decreases in the excretion and $\mathrm{E} / \mathrm{F}$ of sodium occurred.

Potassium excretion (and E/F) in the long and short congestion experiments tended to behave similarly, the excretion varying directly with the glomerular filtration rate and $\mathrm{E} / \mathrm{F}$ showing minor though fairly consistent decreases.

\section{DISCUSSION}

The afferent and efferent mechanisms involved in the renal responses to venous congestion of the limbs reported in this paper are questions of considerable interest. Of greatest interest perhaps is the nature of the stimulus or stimuli calling forth these responses. Since the subjects remained in the supine position throughout the experiments postural stimuli can be eliminated. Of the various remaining possibilities, a reduction in effective circulating blood volume seems one of the more likely. Decreases in urine flow and sodium excretion would appear logically to be homeostatic responses tending to offset any reduction in effective blood volume. Although these responses were most profound when there was collapse of the arterial pressure they could occur before there was any appreciable change in pressure. However, this does not imply that therefore they must have been responses to some other stimulus, since, as previous studies in this laboratory have shown, venous congestion of the limbs always tends to cause arterial pressure to decrease, and does cause it to decrease if the counteracting vasomotor adjustments are blocked (8). It is, therefore, reasonable to suppose that the renal responses to venous congestion of the limbs may be integral parts of the general homeostatic mechanisms for counteracting the hypotensive effects of an inadequate circulating blood volume.

There has been considerable speculation upon the possibility that a decrease in effective circulating blood volume stimulates "volume receptors" to cause salt and water retention of the type de- scribed here. If the term "volume receptor" is meant to connote end-organs sensitive to changes in volume occurring without concomitant changes in pressure, the concept seems totally unrealistic. There are no known sensory end-organs of this type, and indeed it is theoretically impossible to have passive changes in volume of even inelastic vessels that are not associated with at least small changes in pressure. If, on the other hand, "volume receptor" implies that the changes in volume are relatively large as compared with the changes in pressure, the term is acceptable, but seems to add little to our thinking about the problem.

It would appear more fruitful to inquire what and where the changes in pressure may be that could promote reduction in salt or water excretion. Changes in venous, right atrial, right ventricular or pulmonary arterial pressures have all been considered as stimuli possibly capable of producing the renal responses reported in this paper. Studies designed to throw light on these possibilities have been made and have been or will be reported ( 9 , $10,11)$. Suffice it to say here, they do not appear to be very likely possibilities. Neither do stimuli such as discomfort, distention of the veins, loss of fluid into or stretching of the tissues locally in the experimental limbs appear to be responsible (10).

As to the effector mechanisms of the renal responses to venous congestion of the limbs, it appears that they are not dependent upon the splanchnic sympathetic nervous system. As shown in this paper, the responses occur similarly in splanchnicectomized hypertensive patients as in normotensive or unoperated hypertensive subjects. Presumably, therefore, they are mediated at least in part through humoral or local renal mechanisms. The most obvious of the humoral mechanisms that may be considered are the posterior pituitary antidiuretic hormone effects on urine flow, and the adrenal cortical hormone effects on sodium excretion. Studies designed to explore these and other humoral possibilities have also been carried out and will be reported $(6,7)$.

\section{REFERENCES}

1. Wilkins, R. W., Culbertson, J. W., Burrows, B. A., Tinsley, C. M., Judson, W. E., and Burnett, C. H., Antidiuresis and renal vasoconstriction following venous congestion of the limbs in normal, hypertensive, and splanchnicectomized subjects. J. Clin. Invest., 1949, 28, 819. 
2. Brun, C., Knudsen, E. O. E., and Raaschou, F., The influence of posture on the kidney function. I. The fall of the diuresis in the erect posture. Acta med. Scandinav., 1945, 122, 315.

3. Epstein, F. H., Goodyer, A. V. N., Lawrason, F. D., and Relman, A. S., Studies on the antidiuresis of quiet standing: the importance of changes in plasma volume and glomerular filtration rate. J. Clin. Invest., 1951, 30, 63.

4. Goldring, W., and Chasis, $\mathrm{H}$., Hypertension and $\mathrm{Hy}-$ pertensive Disease, The Commonwealth Fund, New York, 1944.

5. Wilson, D. W., and Ball, E. G., A study of the estimation of chloride in blood and serum. J. Biol. Chem., 1928, 79, 221.

6. Judson, W. E., Epstein, F. H., Tinsley, C. M., Burrows, B. A., and Wilkins, R. W., The effects of venous congestion of the limbs upon renal clearances and the excretion of water and salt. II. Studies in patients with diabetes insipidus. To be published.

7. Epstein, F. H., Judson, W. E., Burrows, B. A., and Wilkins, R. W., The effects of venous congestion of the limbs upon renal clearances and the excretion of water and salt. III. Studies in subjects with mannitol-induced diuresis. To be published.

8. Freis, E. D., Stanton, J. R., Finnerty, F. A., Jr., Schnaper, H. W., Johnson, R. L., Rath, C. E., and Wilkins, R. W., The collapse produced by venous congestion of the extremities or by venesection following certain hypotensive agents. J. Clin. Invest., 1951, 30, 435.

9. Epstein, F. H., Post, R. S., and McDowell, M., The effect of an arteriovenous fistula on renal hemodynamics and electrolyte excretion. J. Clin. Invest., 1953, 32, 233.

10. Hatcher, J. D., Judson, W. E., Emerson, C. P., and Wilkins, R. W., The effects of venous congestion of the limbs upon renal clearances and the excretion of water and salt. IV. Prevention in normal subjects by a large transfusion. To be published.

11. Judson, W. E., Hatcher, J. D., Hollander, W., Halperin, M. H., and Wilkins, R. W., The effects of venous congestion of the limbs upon renal clearances and the excretion of water and salt. V. Studies in patients with congestive failure. To be published. 